\title{
Cherenkov Emission Generated by a Modulated Source in 2D Dispersive Photonic Crystal Slabs
}

\author{
Erika Martínez-Sánchez $\mathbb{D}^{1},{ }^{1}$ Gennadiy Burlak $\mathbb{D}^{\mathrm{D}},{ }^{2}$ Carlos Rodríguez-Garcia, ${ }^{3}$ \\ Marisol Gallardo-Heredia, ${ }^{1}$ and Ulises Avila-López ${ }^{1}$ \\ ${ }^{1}$ Facultad de Ingeniería, Universidad Autónoma de Coahuila, Blvd. Fundadores Km, 13 Ciudad Universitaria, Coahuila, \\ Arteaga C. P. 25354, Mexico \\ ${ }^{2}$ Centro de Investigación en Ingeniería y Ciencias Aplicadas, Universidad Autónoma del Estado de Morelos, \\ Av. Universidad 1001, Col. Chamilpa, Cuernavaca, Morelos C.P. 62210, Mexico \\ ${ }^{3}$ Facultad de Ciencias Físico-Matemáticas, Universidad Autónoma de Coahuila, Prol. David Berlanga s/n, \\ Unidad Campo Redondo, Saltillo, Coahuila C.P 25020, Mexico
}

Correspondence should be addressed to Erika Martínez-Sánchez; erika.martinez@uadec.edu.mx

Received 22 October 2019; Revised 16 January 2020; Accepted 31 January 2020; Published 26 February 2020

Academic Editor: Stefano Bellucci

Copyright (C) 2020 Erika Martínez-Sánchez et al. This is an open access article distributed under the Creative Commons Attribution License, which permits unrestricted use, distribution, and reproduction in any medium, provided the original work is properly cited.

\begin{abstract}
This work presents a systematic numerical study of Cherenkov optical radiation generated by a modulated source that moves with uniform velocity on a two-dimensional (2D) photonic crystal (PCr) slab surface. We apply the FDTD technique with emphasis on the dispersion properties of the periodic medium to perform our numerical analysis. The field oscillations generated at the passage of a modulated source in the PCr produce a series of spectral resonances corresponding to the eigenmodes in the spatial frequency domain for the photonic slab. The amplitudes of the field oscillations have maximal values in the group cone closely to the path of the moving charge.
\end{abstract}

\section{Introduction}

Dielectric two-dimensional photonic crystals, composed of either dielectric rods or air columns, are widely used to obtain a high control over the propagation of light without loss of energy. It has been shown theoretically and experimentally that photonic crystal slab structural defects behave as waveguides [1], resonant devices [2], nanocavity laser [3], photonic circuits, and various functional devices [4-6]. In a conventional material, the Cherenkov radiation is coherent, and the charged particle movement is associated with a velocity threshold, a forward-pointing radiation cone, and a forward direction of emission $[7,8]$. Cherenkov radiation generated by a charged particle in dielectric homogeneous medium has been extensively studied. In a more complex periodic medium, e.g., a meta-material, we can also get a variety of anomalous effects [9], such as backward direction Cherenkov radiation [10-15]. However, the Cherenkov radiation (CR) has a different behavior in a photonic crystal. Photonic crystals, where very complex Bragg scattering is possible, exhibit a new medium for unusual radiation pattern under different particle-velocity regimes. [16-19].

There are various approaches to study the influence of a periodic medium on the Cherenkov effect. For example, in [20], there is a complete description of stimulated Cherenkov radiation and laser oscillation in a photonic crystal in the range $10 \mathrm{GHz}$, getting significant power and high spectral and spatial coherence [20]. The fiber-optical Cherenkov radiation also was of great interest in recent years $[17,21]$.

Other studies are devoted to the analysis of the Cherenkov emission spectrum through the photonic band structures calculation in photonic crystals. In such simulations, the method of the finite differences in time domain (FDTD) was applied, and it was shown that the spectral and spatial distributions can be varied over different ranges of velocities $[16,22]$. 
However, in the aforementioned studies, the case of a modulated source moving through a slab of photonic crystal has not been studied. We propose a simpler approach based on the geometrical design of the photonic crystal and characteristics of the source, allowing obtaining a coherent radiation in the specific frequency ranges. Unlike previous works, our approach does not require an analysis of the crystal band structure. Getting the output of desired wavelengths is of high interest for creation of novel light sources, e.g., in the extreme ultraviolet (EUV) radiation range [23]. Among other applications, such a study includes the velocity-sensitive particle detection and radiation generation at selected range of frequencies.

Askar'yan demonstrates theoretically that Cherenkov radiation generated from a modulated source moving with velocity $v_{0}$ occurs when the medium has an inhomogeneous structure. For example, when the wave packet is transmitted through the boundary between two media, then the transition radiation should be produced [24]. It is interesting to investigate the optical modes in the photonic crystal associated with the Cherenkov radiation generated by a modulated wave train, since this kind of wave packet is produced in most cases of practical interest [24].

In this paper, we study numerically the Cherenkov optical radiation in a two-dimension (2D) photonic crystal slab (PCr). The modulated source is nonrelativistic, having the eigenfrequency $\omega_{0}$. We performed the finite differences time domain (FDTD) simulations [25], where the size of the photonic crystal structure is fixed, and the values of dielectric permittivity inside and outside the holes of the structure are varied. With the use of spectral Fast Fourier Transform (FFT) analysis we have found that the spatial frequencies of the oscillations produced by Cherenkov emission also oscillates correspondingly to the eigenmodes located in the gap zone of the photonic crystal slab.

\section{Basic Equations}

In this paper, we exploit the FDTD 3D algorithm that became a widely used algorithm for modelling in complex materials. The Maxwell equations are

$$
\begin{aligned}
& \nabla \times \mathbf{E}(\mathbf{r}, t)=-\mu_{0} \frac{\partial}{\partial t} \mathbf{H}(\mathbf{r}, t) \\
& \nabla \times \mathbf{H}(\mathbf{r}, t)=\varepsilon_{0} \varepsilon \frac{\partial}{\partial t} \mathbf{E}(\mathbf{r}, t)+q \mathbf{v}_{0} f(\mathbf{r}, t),
\end{aligned}
$$

where $\varepsilon=\varepsilon(\mathbf{r})$ is a dielectric permittivity of the PCr structure [26]. We consider the charge $q$ that moves with uniform velocity $\mathbf{v}_{0}$ parallel to $y$ direction: $\mathbf{v}_{0} \| \widehat{e}_{y}$, closely to the $\langle x, y\rangle$ surface. In the numerical grid, the point particle (bunch) is simulated by the Gaussian impulse $f(r, t)=\cos \left(\omega_{0} t\right)$ $W^{-3} \exp \left\{-\left[x^{2}+\left(y-v_{0} t\right)^{2}+z^{2}\right] / W^{2}\right\}$, where $\omega_{0}$ is the modulating frequency and $W$ is the width.

To perform the numerical simulations, we used the dimensionless variables for normalization, the vacuum light velocity $c=\left(\varepsilon_{0} \mu_{0}\right)^{-0.5}$, and the typical spatial scale for nanooptic objects $l_{0}=500 \mathrm{~nm}$ were used. The electrical and magnetic fields are renormalized with the electrical scale
$E_{0}=q l_{0} \varepsilon_{0}$, and magnetic scale $H_{0}=\left(\varepsilon_{0} / \mu_{0}\right)^{0.5} E_{0}$, respectively.

\section{Numerical Results}

The considered photonic crystal slab structure perforated by periodical air rods in a dielectric medium is shown in Figure 1.

2D photonic crystals have been extensively studied because they are relatively easier to fabricate using existing techniques and to operate in optical frequencies [27-30]. We focus our attention on the 2D lattice of air holes that is a widely used structure, since the triangular lattice of air holes has a large band gap [31], see also experiments [32,33]. The slab thickness, radius, and refractive index contrast determine the properties of photonic crystal slabs [34]. In our approach, we use a high index contrast, that is required between the dielectric material, and the holes to open a bandgap in the xy-plane [33]. Since the lattice constant of the photonic crystal must be comparable to the wavelength of the light [34], we use, $a=500 \mathrm{~nm}$. The radius and height of the holes we have chosen are in the experimentally accessible range for reasonable values $[31,35,36]$, such that, $r=0.3$ and $h=1 a$, which normally is used within the resolution range of electron beam lithography. Such parameter values also allow improving the accuracy of numerical simulation, [31]. We should note that, since the spatial period of $\mathrm{PCr}$ is comparable with the field wavelength $\left(l_{0} / \lambda\right) \leq 1$, such a $2 \mathrm{D}$ system acts as a distributed $2 \mathrm{D}$ plane resonator having a special eigenfrequency spectrum.

Since the refractive index of a photonic system varies in space and it is well-defined only locally (or in average), we only know numerical methods to calculate the Cherenkov emission in such 3D inhomogeneous photonic systems. To realize the FDTD simulation, the scheme from [2] was followed, which we have elaborated for our purposes. The renormalized variables were used $(\mathrm{d} x / \mathrm{d} t) \longrightarrow 3(\mathrm{~d} x / \mathrm{d} t)=$ $(\sqrt{3}(\mathrm{~d} l / \mathrm{d} t))=c_{n} \equiv 1.73$ that is the vacuum light velocity in the numerical approach, such that $v_{0}<c_{n}$, where $v_{0}$ is the velocity of charge.

3.1. Cherenkov Radiation in a Photonic Crystal Slab. The practically interesting case of the Cherenkov radiation (CR) in the case of dielectric permittivity of the slab and holes with distinct values $\varepsilon_{s} \neq \varepsilon_{h}$ was considered. Besides, the critical velocity of the Cherenkov radiation in a homogeneous medium was used as a reference value.

Figure 2 shows the field oscillations within the Cherenkov cone $v_{0}=1.5$ generated at the time when the particle touches the output boundary of the computational system. Figure 2(a) shows the $3 \mathrm{D}$ field component $\left|E_{x}\right|$, with the uses radius $r=0.25$ and $\varepsilon_{s}=3, \varepsilon_{h}=1$ as permittivity of slab and holes, respectively. Figure 2(b) displays the field $\left|E_{x}\right|$ when $r=0.35$ and $\varepsilon_{s}=15$. It is worth noting that the increase in hole radius does not significantly affect the field of Cherenkov radiation. However, when the difference in dielectric constants is increased, the angle of the cone is reduced, but the amplitude of oscillations becomes larger. We observe 


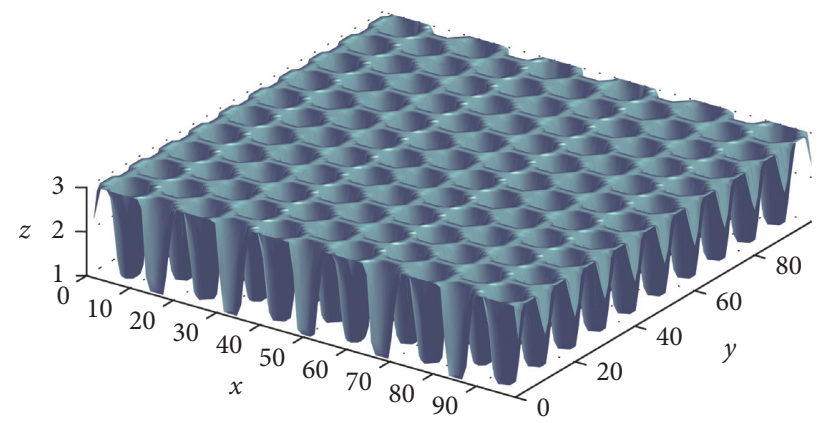

Figure 1: No magnetic photonic crystal slab structure with average dielectric permittivity, where dielectric slab $\left(\varepsilon_{s}=3\right)$ is perforated by holes $\left(\varepsilon_{h}=3\right)$ ordered periodically.

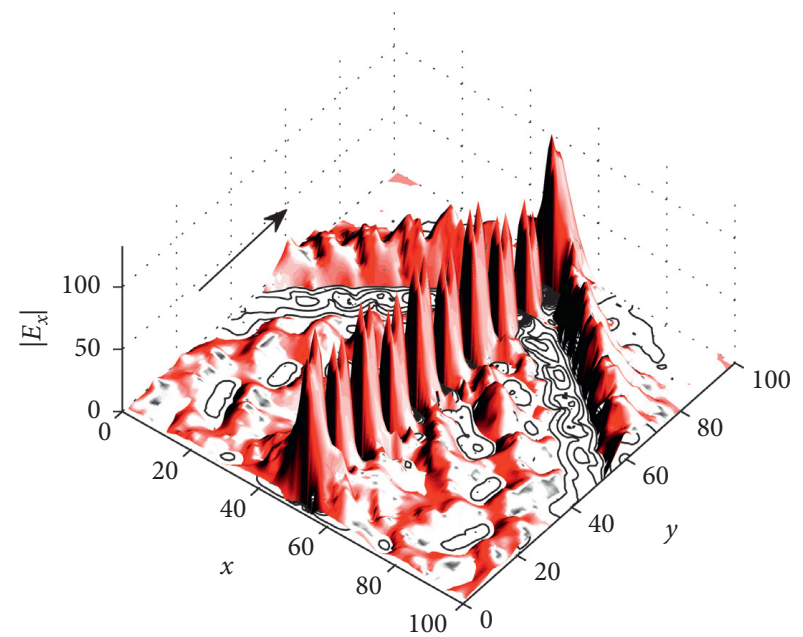

(a)

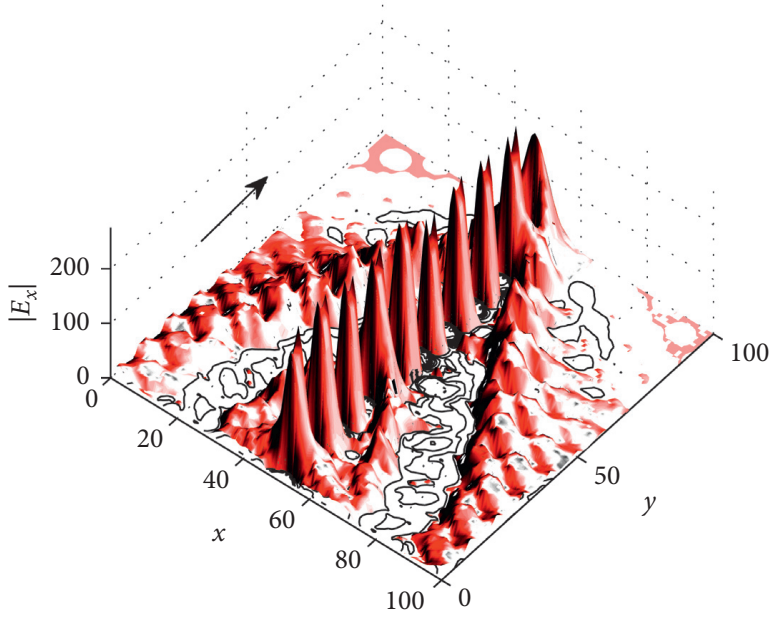

(b)

Figure 2: (Color on line) snapshots the field $\left|E_{x}\right|(x, y)$ in plane $(x, y)$ for a particle moving with $v_{0}=1.5>v_{c}$ and frequency source $\omega_{0}=0$. (a) $r=0.25, \varepsilon_{s}=3$. (b) $r=0.35, \varepsilon_{s}=15$. The Cherenkov group cone is well defined with oscillations in the path of the charge.

that such a geometry on the field within the Cherenkov cone acquires a highly inhomogeneous structure due to the discontinuity of dielectric permittivity in the boundary of holes.

Cherenkov radiation (CR) in a photonic crystal arises from a coherent excitation of its eigenmodes by the moving charge. Its origin lies in both the transition radiation, which occurs when the charge crosses a dielectric boundary or experiences at an inhomogeneous dielectric environment, and the conventional CR. In these cases, the coherence is preserved throughout the medium as we see in [37]. Unlike the case of Cherenkov radiation in a homogeneous medium, there exist radiating modes in this inhomogeneous dielectric case. It is interesting to study the behavior of these oscillations within the Cherenkov group cone [38].

A simple physical interpretation of the group cone can be put forward in terms of group velocity considering that for each direction around the charge velocity, the burst of Cherenkov light is emitted into a group of modes, while the peak of the pulse moves in space with a velocity equal to the group velocity $v_{g}$ experiencing an almost negligible absorption [38].
In the following, the spectra of electromagnetic oscillations associated with the Cherenkov emission for different perforated slab systems are calculated.

In Figure 3 the values of optical eigenmodes of $\mathrm{PCr}$ excited by the moving charge calculated for different $\mathrm{PCr}$ configurations given by variation in the dielectric permittivity of slab $\left(\varepsilon_{s}\right)$ and cases of radiuses $r=0.25 ; 0.3 ; 0.35$ are displayed. A decreasing value of the spectral peak's values for greater contrast of the permittivities is observed. The inset panel of Figure 3 shows the curve of the set of spatial frequencies obtained as a result of our numerical simulations (red line) and the values by applying the formula $f=(c / \lambda) \sqrt{\varepsilon_{p}}$ (blue line), which is a simple dimension relationship, where $\varepsilon_{p}$ is a weighted dielectric permittivity of PCr $\varepsilon_{p}=\left(\varepsilon_{s}\left(V_{s}-V_{c}\right)-\varepsilon_{h} V_{c}\right) /\left(V_{s}+V_{c}\right)$ [37]. Here, $V_{s}$ and $V_{c}$ are the volume of the slab and holes, respectively.

3.2. Modulated Source for a Hole Slab of Photonic Crystal. Uniform motion of a modulated source in the medium can produce radiation when the medium is inhomogeneous, for example, when the wave packet is transmitted through the boundary between two media, then transition radiation 


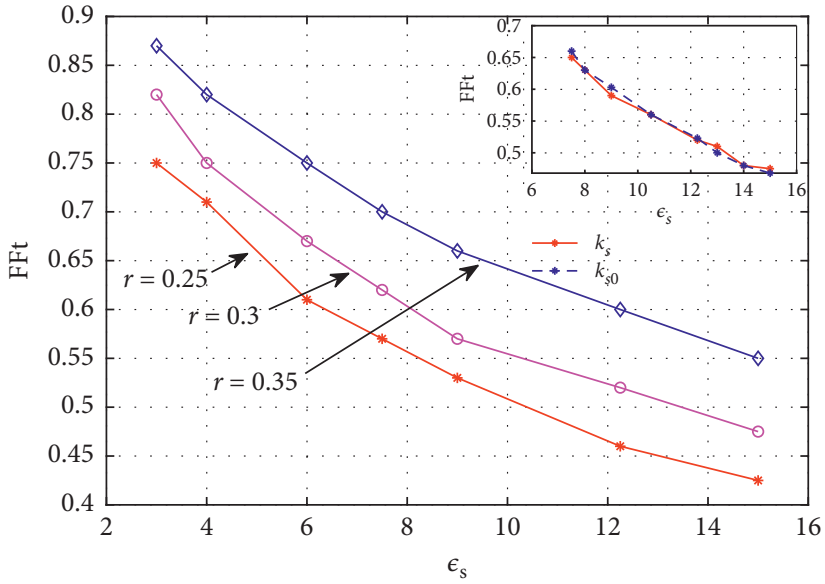

FiguRe 3: Frequency $\left(k_{s}\right)$ calculated numerically for different values of $\varepsilon_{s}$ in a slab of holes $\varepsilon_{h}=1$ for radius $r=0.25,0.3$ and 0.35 . It is seen that for a greater contrast of the dielectric constant, the value of the $k_{s}$ decreases, until reaching the minimum value in $k_{s} \sim 0.43$ for $r=0.25$ and the maximum $k_{s} \sim 0.87$ for $r=0.35$. The graph inside the panels show the case of $k_{s}$ for $r=0.3$ where the red curve is the experimental data, while the blue one shows the results following from the equation $\varepsilon_{p}=\left(\varepsilon_{s}\left(V_{s}-V_{c}\right)-\varepsilon_{h} V_{c}\right) /\left(V_{s}+V_{c}\right)$. See more details in text.

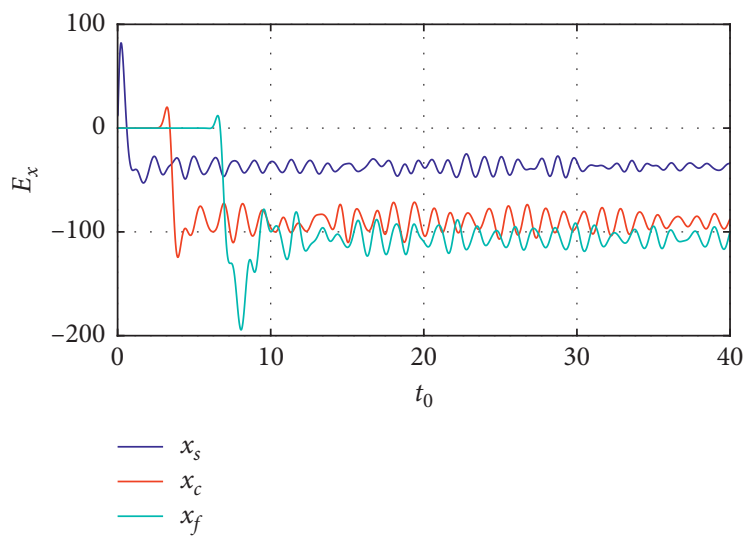

(a)

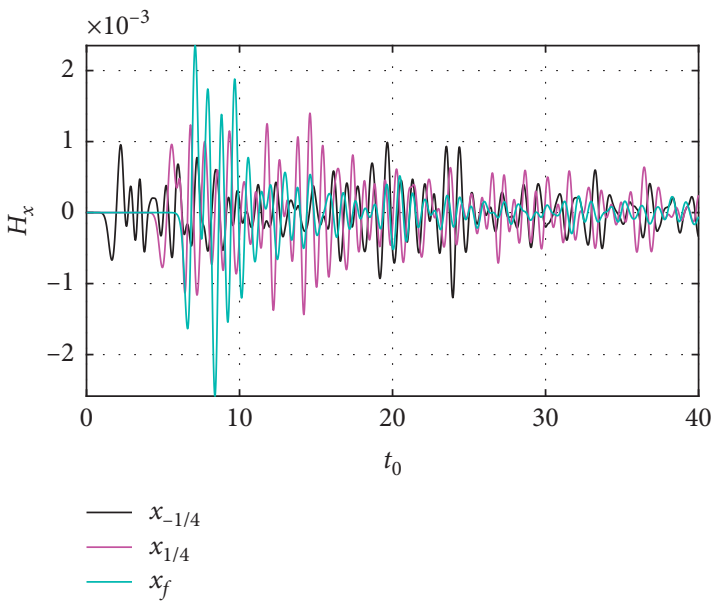

(c)

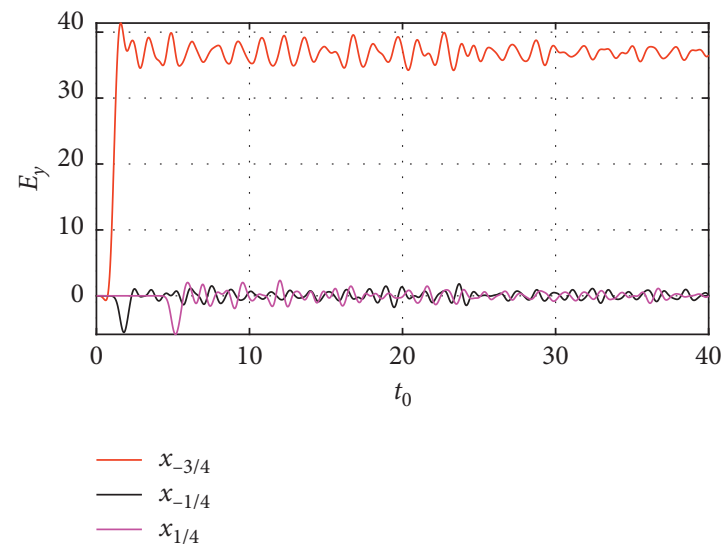

(b)

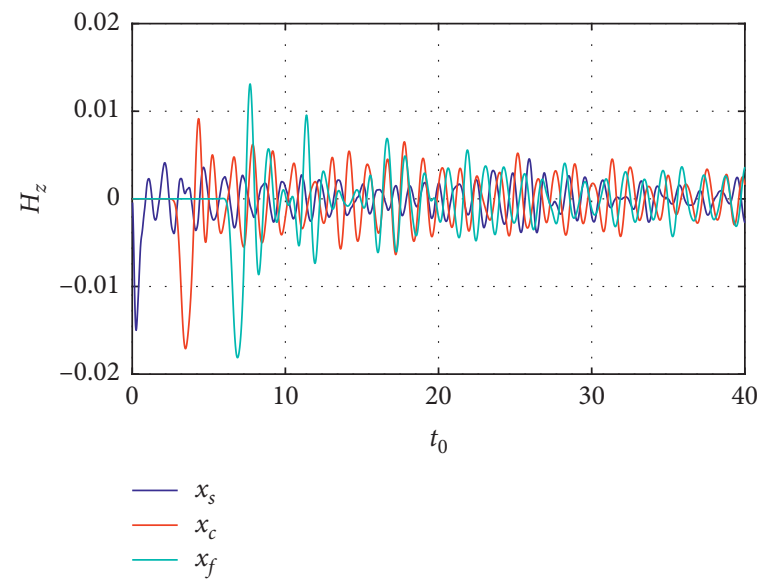

(d)

Figure 4: The time dependence for the components (a) $E_{x}$, (b) $E_{y}$, (c) $H_{x}$, and (d) $H_{z}$, in various points along the charge path with velocity $v_{0}=1.5$ and frequency $\omega_{0}=0$. 


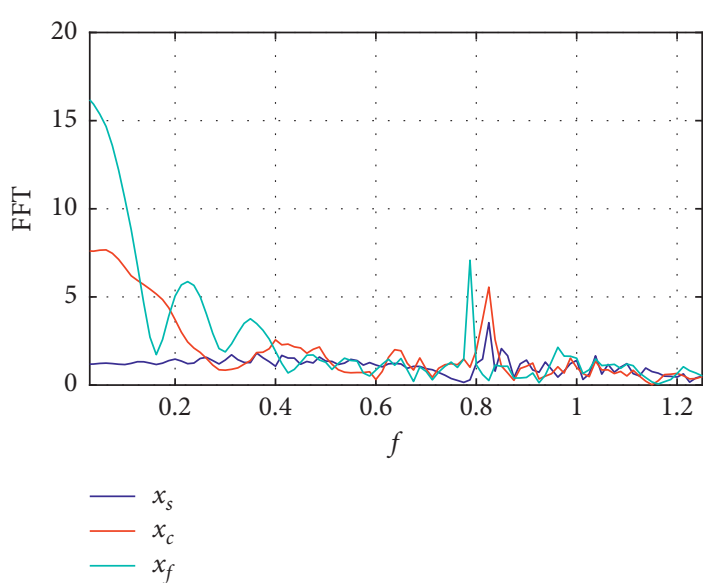

(a)

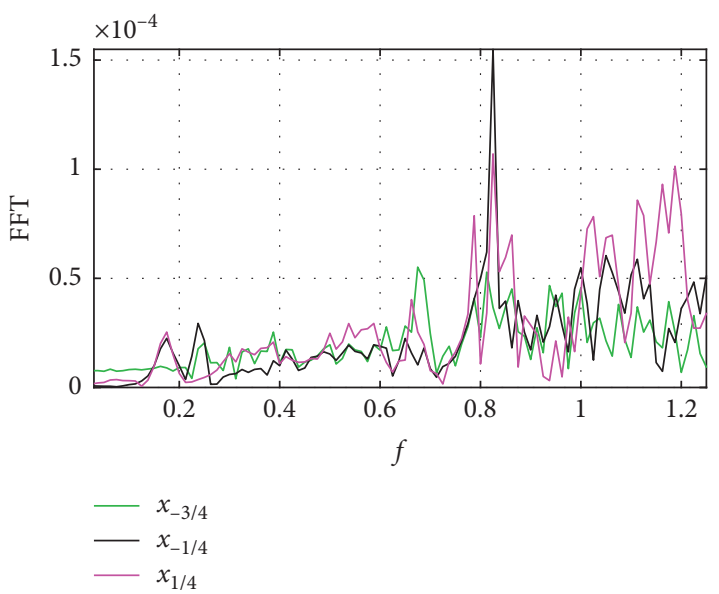

(c)

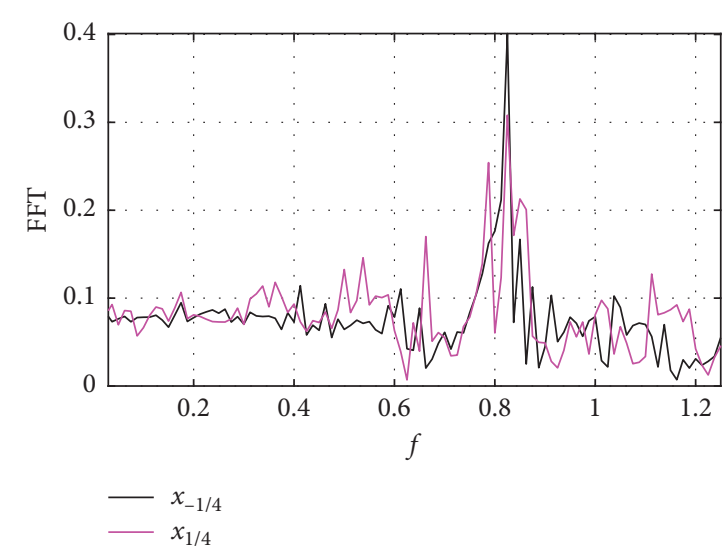

(b)

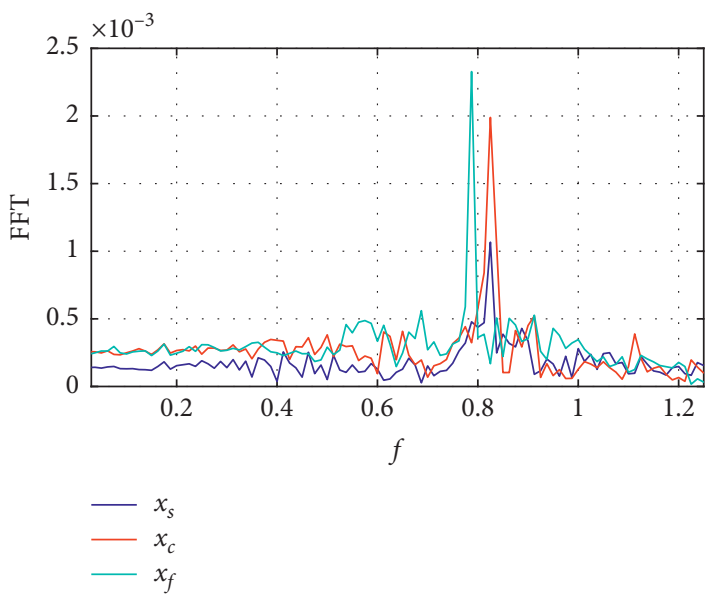

(d)

Figure 5: (Color on line) the spatial frequency spectrum of electromagnetic oscillations shown in Figure 4 for various points along the charge path, having highest spectral peaks at $k_{s} \sim 0.82$. Such oscillations correspond to optical eigenmodes of PCr excited by the moving charged particle for components (a) $E_{x}$, (b) $E_{y}$, (c) $H_{x}$, and (d) $H_{z}$, for values $v_{0}=1.5$ and $\omega_{0}=0$.

should be created [24]. In the following, we analyze in more detail the Cherenkov field for a modulated source with $\omega_{0}=$ 0 and $\omega_{0} \neq 0$. A geometry with $r=0.3$ and a permittivity of the slab $\varepsilon_{s}=3$ were studied.

Figure 4 shows the time dependence of field at a longtime simulation when a particle with velocity $v=1.5$ and modulation $\omega_{0}=0$ passes over a slab. The Figures 4(a) and 4(b) display the components $E_{x}$ and $E_{y}$, respectively. We observe that $E_{x, y}$ amplitudes have an oscillating shape shift with a pedestal defined by a point position. Panel (c), (d) shows that the field $H_{y, z}(x, y)$ has smoother shape due to the continuity of magnetic field in the system.

Figure 5 displays the frequency spectrum of electromagnetic oscillations shown in Figure 4 for various points along the charged path, having the highest spectral peaks at $k_{s} \sim 0.82$. Such oscillations correspond to optical eigenmodes of $\mathrm{PCr}$ excited by the moving charged particle for components (a) $E_{x}$, (b) $E_{y}$, (c) $H_{x}$, and (d) $H_{z}$ for values $v_{0}=1.5$ and $\omega_{0}=0$.

Figure 6 shows the 3D spatial structure of the field $E_{x}$ at long-time simulation $L^{2}=10 l_{0} \times 10 l_{0}$, where $l_{0}=500 \mathrm{~nm}$.
The cases (a) $v_{0}=1.5, \omega_{0}=0$; (b) $v_{0}=1.5, \omega_{0}=0.82$; (c) $v_{0}=1, \omega_{0}=0$; (d) $v_{0}=1, \omega_{0}=0.82$ are displayed. Figure 6 shows that the distribution of the field $E_{x}$ in $\mathrm{PCr}$ is structurally stable, and this is due to discontinuity in the boundary of holes, for all cases shown.

Figure 7 , shows the frequency spectrum of electromagnetic oscillations corresponding to Figures 6(c) and 6(d), for various points along the charge path at $x_{ \pm(1 / 4)}, x_{c}, x_{3 / 4}, x_{s}, x_{f}$ at $x= \pm(L / 4,0)$. In Figure $7(\mathrm{a})$, we observe the existence of near-static, no radiating field artificial peak around $k_{s}=0$ [38], in panel (b), $k_{s} \simeq 0.82$, and such oscillations correspond to optical eigenmodes in $\mathrm{PCr}$ excited by moving charged particle; this evince the existence of the Cherenkov radiant field for a modulated source in an inhomogeneous medium.

Figure 8(a) shows the characteristic Cherenkov cone when $\omega_{0}=0$, and $v_{0}=1.5$. In Figure 8(b), the group cone is well defined but with fewer oscillations in the charged path, this is due to the modulation of the particle bunch. The situation changes when the velocity of the source is decreased. In this case, when $v_{0}=1$, we do not observe the 


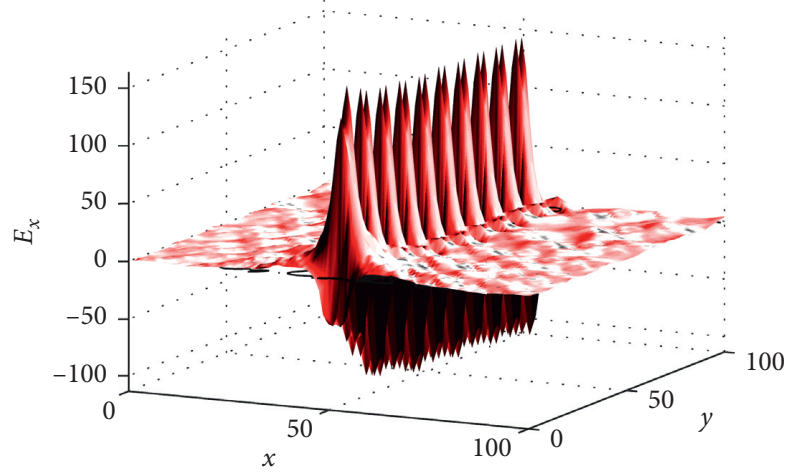

(a)

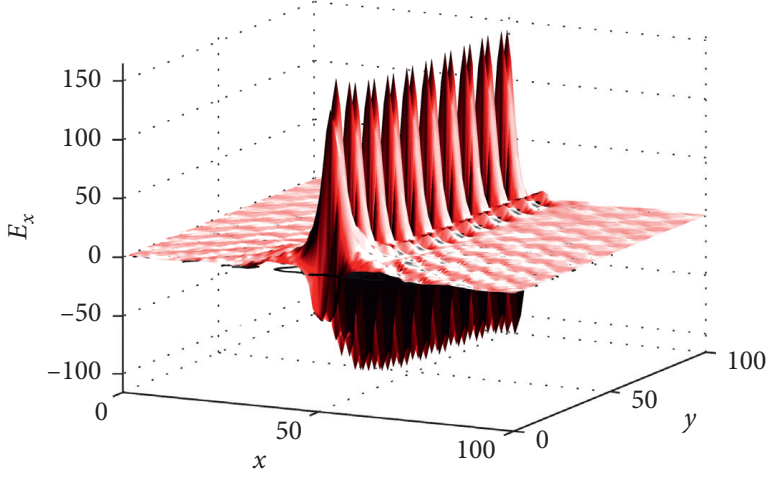

(c)

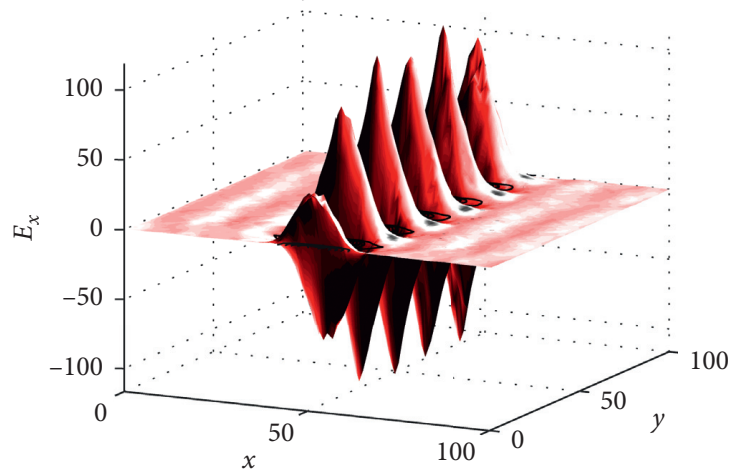

(b)

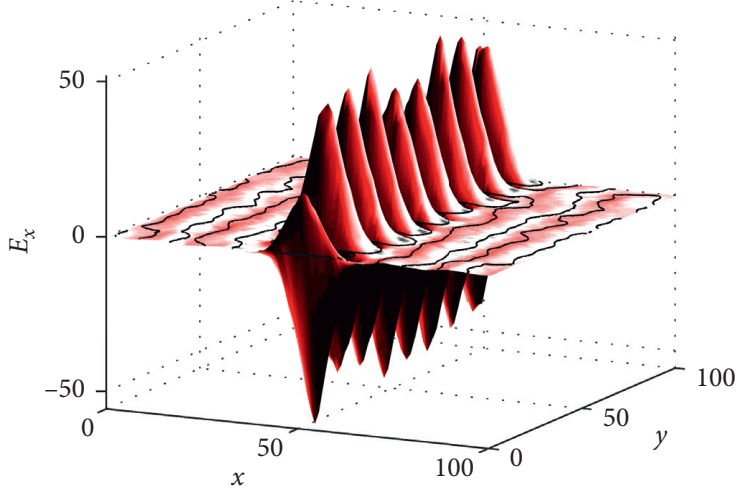

(d)

Figure 6: (Color on line) the spatial $(x, y)$ shape of field component $E_{x}$ for particle that moves at different speeds and modulated frequencies. (a) $v_{0}=1.5, \omega_{0}=0$. (b) $v_{0}=1.5, \omega_{0}=0.82$. (c) $v_{0}=1, \omega_{0}=0$. (d) $v_{0}=1, \omega_{0}=0.82$. (a), (c) Oscillations are very similar when $\omega_{0}=0$, and we observe the same amplitude. (b), (d) The oscillations do not correspond to the positions of the holes in the structure, and this is due to the modulation of the source. The number of oscillations and amplitude of $E_{x}$ has dependence of $\omega_{0} \neq 0$.

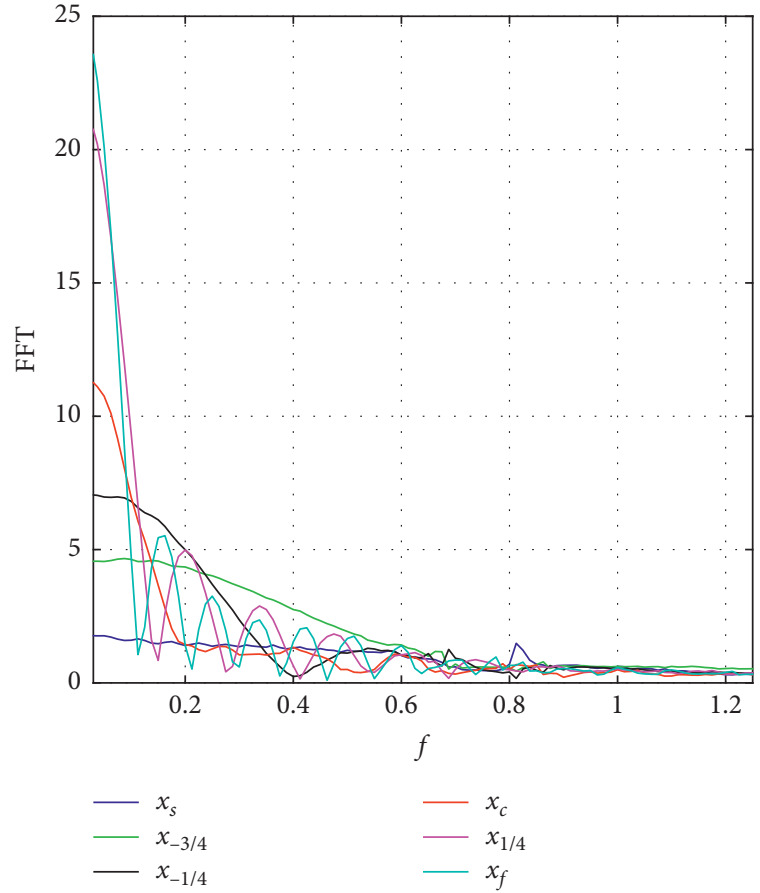

(a)

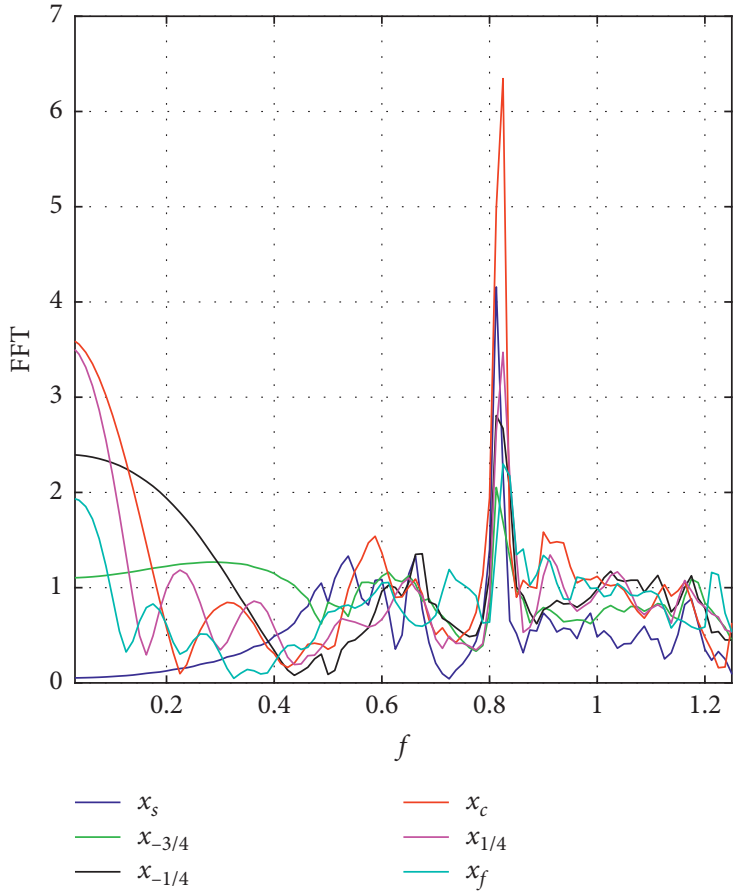

(b)

FiguRE 7: Fourier spectrum (wavenumbers domain) for (a) $v_{0}=1, \omega_{0}=0$. (b) $v_{0}=1, \omega_{0}=0.82$. In panel (a) we observe the existence of nearstatic no radiating field artificial peak around $k_{s}=0$ while that of (b) well-defined peaks $k_{s} \simeq 0.82$ corresponds to optical eigenmodes of PCr. 


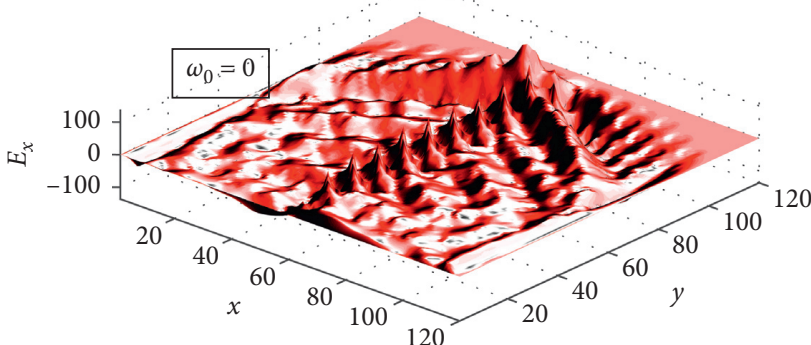

(a)

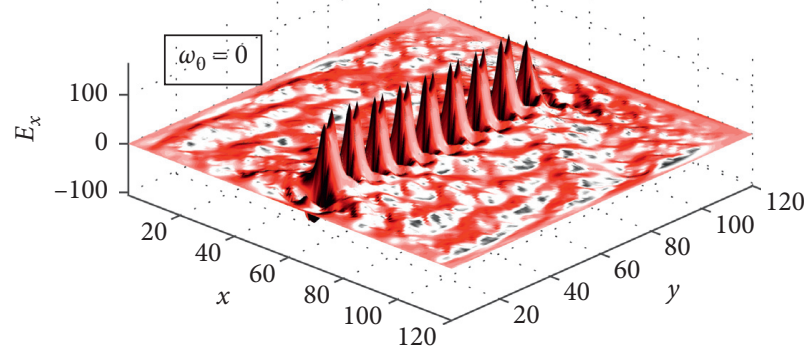

(c)

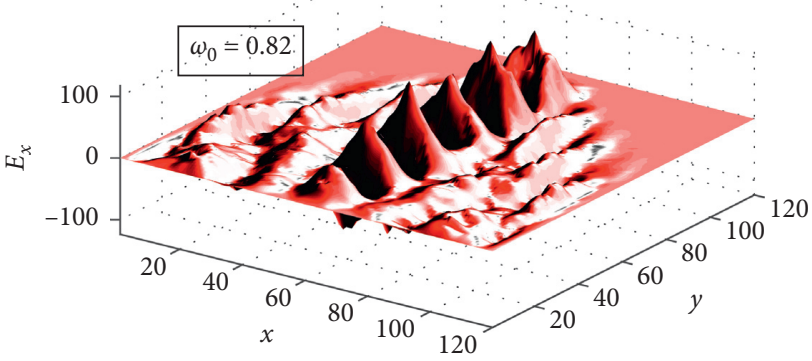

(b)

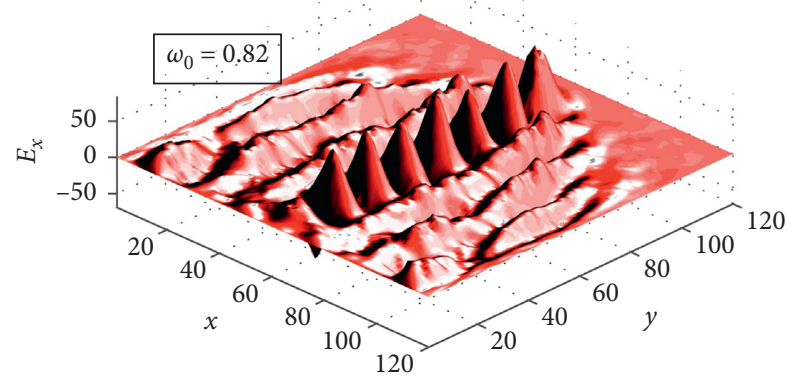

(d)

Figure 8: (Color on line) snapshots the field $E_{x}(x, y)$ in plane $(x, y)$ for particle moving with (a)-(b) $v_{0}=1.5$ and (c)-(d) $v_{0}=1$. (a) Field $E_{x}$ for $\omega_{0}=0$, the Cherenkov cone is well defined with oscillations in the path of the charge. (b) Field $E_{x}$ for $\omega_{0}=0.82$, the Cherenkov emission is observed, with a lower number of oscillations than subsection (a) due to the modulation of the source. (c) $\omega_{0}=0$, oscillations in the path of the charge; however, in this case, we did not observe the group cone. (d) Cherenkov group cone for $\omega_{0}=0.82$ and $v_{0}=1$.

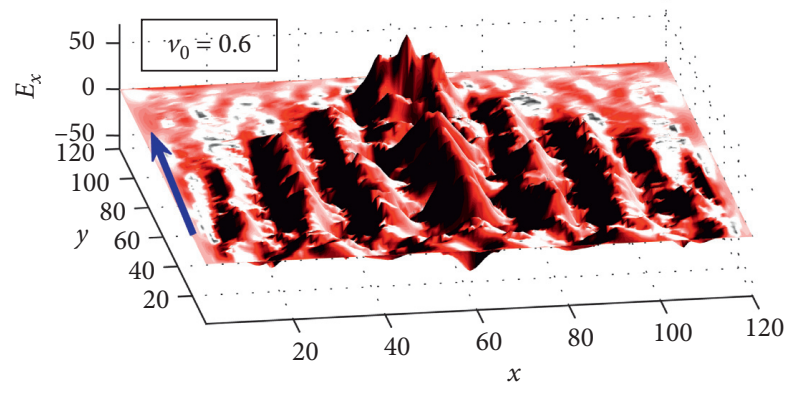

(a)

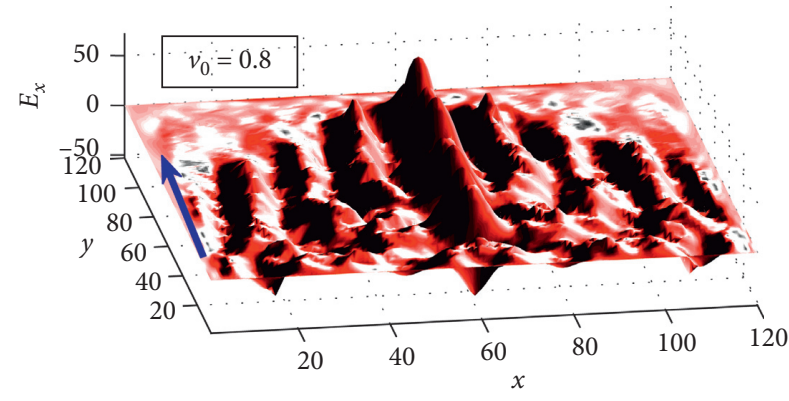

(c)

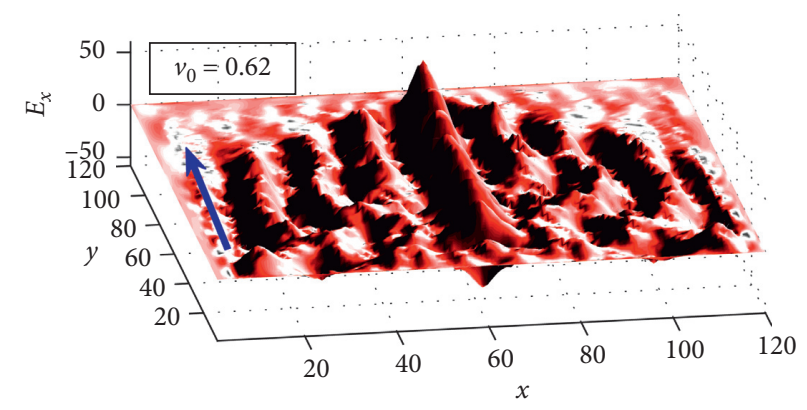

(b)

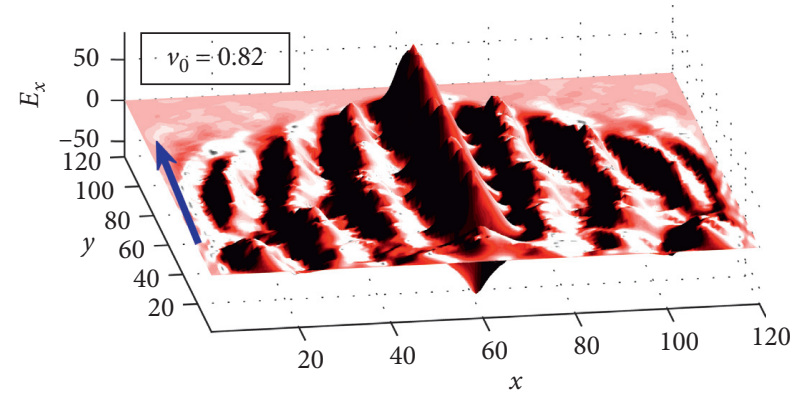

(d)

FIGURE 9: (Color on line) snapshots the field $E_{x}(x, y)$ in plane $(x, y)$ for particle bunch moving with parameters (a) $\varepsilon_{s}=12.25, v_{0}=0.6, \omega_{0}=0.52$; (b) $\varepsilon_{s}=9, v_{0}=0.62, \omega_{0}=0.57$; (c) $\varepsilon_{s}=6, v_{0}=0.8, \omega_{0}=0.67$; (d) $\varepsilon_{s}=3, v_{0}=0.1, \omega_{0}=0.82$. The Cherenkov wave cone is well defined for all cases shown. 
Cherenkov group cone (see Figure 8(c)). In periodic dispersive materials, the critical velocity in the medium is not well defined. We observe that it is possible to extend the velocity range $v_{0}$ and observe the group cone for a modulated source, and this situation is shown in Figure 8(d).

Figure 9 shows the group cone for different $\mathrm{PCr}$ systems, when $\omega_{0} \sim k_{s}$. Cases are shown: (a) $\varepsilon_{s}=12.25, \omega_{0}=0.52$; (b) $\varepsilon_{s}=9, \omega_{0}=0.57$; (c) $\varepsilon_{s}=6, \omega_{0}=0.67$; (d) $\varepsilon_{s}=3, \omega_{0}=0.82$. Cherenkov group cone is well defined for all cases.

\section{Conclusions}

The optical field of Cherenkov by a modulated source in a perforated slab of photonic crystal is studied with details. Similar field structures can be produced in various cases of practical interest; therefore, we can conclude that considered periodic medium with studied optical properties may by adequate to this scope. We have shown that the Cherenkov group cone exists when the modulation frequency $\omega_{0} \neq 0$ in a wider range of charge velocities. In our approach, the slab parameters are fixed, while the spatial frequency spectrum is a result of numerical simulations. Such oscillations of the Cherenkov field correspond to optical eigenmodes of the photonic crystal excited by the moving modulated source. Applications may include velocity-sensitive particle detectors, laser oscillation, fiber-optic Cherenkov, detection, and field radiation generation at selectable frequency ranges.

\section{Data Availability}

No data were used to support this study.

\section{Conflicts of Interest}

The authors declare that they have no conflicts of interest.

\section{References}

[1] P. S. J. Russell, D. M. Atkin, T. A. Birks, and P. J. Roberts, "Bound modes of two-dimensional photonic crystal waveguides," Microcavities and Photonic Bandgaps: Physics and Applications, vol. 324, pp. 203-218, 1996.

[2] Se-H. Kim, S.-K. Kim, and Y.-H. Lee, "Vertical beaming of wavelength-scale photonic crystal resonators," Physical Review B, vol. 73, Article ID 235117, 2006.

[3] O. Painter, R. K. Lee, Scherer et al., "Two-dimensional photonic band-gap defect mode laser," Science, vol. 284, no. 5421, pp. 1819-1821, 1999.

[4] G. Steven, S. F. Johnson et al., "Guided modes in photonic crystal slab," Physical Review B, vol. 60, p. 5751, 1999.

[5] K. Sakoda, Optical Properties of Photonic Crystals, Springer Berlin Heidelberg, New York, NY, USA, 2005.

[6] JD. Joannopoulos, SG. Johnson, JN. Winn et al., Photonic Crystals Molding the Flow of Light, Princeton University Press, Princeton, NJ, USA, 2008.

[7] PA. Cherenkov, "Visible emission of clean liquids by action of $\gamma$-radiation," Doklady Akademii Nauk, vol. 2, pp. 451-454, 1934.

[8] GN. Afanasiev, "Cherenkov radiation in a dispersive medium: Vavilov-Cherenkov and synchrotron radiation," in Fundamental Theories of Physics, Kluwer Academic Publishers, Dordrecht, Netherlands, 2004.
[9] G. Burlak and E. Martinez-Sanchez, "Change of structure of the Cherenkov emission at modulated source in dispersive metamaterials," Progress In Electromagnetics Research, vol. 139, pp. 277-288, 2013.

[10] O. A. Yu and V. M. Yakovenko, "Cherenkov radiation by an electron particle that moves in a vacuum above a left-handed material," Physical Review B, vol. 79, no. 20, pp. 193402193412, 2005.

[11] Z.-Y. Duan, B.-I. Wu, S. Xi, H. Chen, and M. Chen, "Research progress in reversed Cherenkov radiation in double-negative metamaterials," Progress in Electromagnetics Research, vol. 90, pp. 75-87, 2009.

[12] S. Xi, H. Chen, T. Jiang et al., "Experimental verification of reversed Cherenkov radiation in left-handed metamaterial," Physical Review Letters, vol. 103, Article ID 194801, 2009.

[13] G. Burlak, "Spectrum of Cherenkov radiation in dispersive metamaterials with negative refraction index," Progress in Electromagnetics Research, vol. 132, pp. 149-158, 2012.

[14] J. Zhou, Z. Duan, Y. Zhang et al., "Numerical investigation of Cherenkov radiations emitted by an electron beam bunch in isotropic double-negative metamaterials," Nuclear Instruments and Methods in Physics Research Section A: Accelerators, Spectrometers, Detectors and Associated Equipment, vol. 654, no. 1, pp. 475-480, 2011.

[15] Z. Duan, C. Guo, and M. Chen, "Enhanced reversed Cherenkov radiation in a waveguide with double-negative metamaterials," Optics Express, vol. 19, no. 15, pp. 13825-13830, 2011.

[16] C. Luo, M. Ibanescu, S. G. Johnson et al., "Cerenkov radiation in photonic crystals," Science, vol. 299, no. 5605, pp. 368-371, 2003.

[17] G. Chang, L.-J. Chen, and F. X. Kärtner, "Highly efficient Cherenkov radiation in photonic crystal fibers for broadband visible wavelength generation," Optics Letters, vol. 35, no. 14, pp. 2361-2363, 2010.

[18] S. Xiang-Wei, Y. Jin-Hui, K. Wang et al., "Highly efficient Cherenkov radiation generation in the irregular point of hollow-core photonic crystal fiber," Chinese Physics B, vol. 21, no. 11, Article ID 114102, 2012.

[19] F. J. García de Abajo, A. G. Pattantyus-Abraham, N. Zabala et al., "Cherenkov effect as a probe of photonic nanostructures," Physical Review Letters, vol. 91, Article ID 143902, 2003.

[20] T. Denis, M. W. van Dijk, J. H. H. Lee et al., "Coherent Cherenkov radiation and laser oscillation in a photonic cristal," Physical Review A, vol. 94, no. 5, 2016.

[21] G. Chang, Li-J. Chen, and X. Franz, "Kartner "Fiber-optic Cherenkov radiation in the few-cycle regime"," Optics Express, vol. 19, no. 7, 2011.

[22] C. Kremers, D. N. Chigrin, and J. Kroha, "Theory of Cherenkov radiation in periodic dielectric media: emission spectrum," Physical Review A, vol. 79, 2009.

[23] J. M. Andre, C. Bonnelle, and B. Pardo, "Tunable and bright EUV sources using the interaction of medium-energy electron beams with periodic multilayer targets," Nuclear Instruments and Methods in Physics Research Section A: Accelerators, Spectrometers, Detectors and Associated Equipment, vol. 498, no. 1-3, 532 pages, 2003.

[24] GA. Askar'yan, "Cerenkov radiation and transition radiation from electromagnetic waves," Journal of Experimental and Theoretical Physics, vol. 42, pp. 1360-1364, 1962.

[25] A. Taflove and S. C. Hagness, Computational Electrodynamics: The Finite-Difference Time-Domain Method, Artech House, Boston, MA, USA, 2005. 
[26] J. D. Jackson, Classical Electrodynamics, John Willey and Sons, Hoboken, NJ, USA, 1998.

[27] R. K. Lee, O. J. Painter, B. Kitzke, A. Scherer, and A. Yariv, "Photonic bandgap disk laser," Electronics Letters, vol. 35, no. 7, pp. 569-570, 1999.

[28] P. R. Villeneuve, S. Fan, S. G. Johnson, and J. D. Joannopoulos, "Three-dimensional photon confinement in photonic crystals of low-dimensional periodicity, Photonic crystals and microstructures," IEE Proceedings-Optoelectronics, vol. 145, no. 6, 1998.

[29] S. Fan, P. R. Villeneuve, and J. D. Joannopoulos, "High extraction efficiency of spontaneous emission from slabs of photonic crystals," Physical Review Letters, vol. 78, no. 17, 1997.

[30] Jelena Vuckovic, O. Painter, Y. Xu, A. Yariv, and A. Scherer, "Finite-difference time-domain calculation of the spontaneous emission coupling factor in optical microcavities," IEEE Journal of Quantum Electronics, vol. 35, no. 8, 1999.

[31] A. Scherer, O. Painter, B. D'Urso, R. Lee, and A. Yariv, "InGaAsP photonic band gap crystal membrane microresonators," Journal of Vacuum Science \& Technology B, vol. 16, 1998.

[32] I. Masahiro, N. Susumu, A. Chutinan, and T. Tokuda, "Coherent two-dimensional lasing action in Surface-emitting laser with triangular-lattice photonic crystal structure," Applied Physics Letters, vol. 75, no. 3, pp. 316-318, 1999.

[33] S. G. Johnson, S. Fan, P. R. Villeneuve, and J. D. Joannopoulos, "Guided modes in photonic crystal slabs," Physical Review B, vol. 60, no. 8, 1999.

[34] J. R. Joannopoulos Meade and J. Winn, "Photonic Crystals", Princeton Press, Princeton, NJ, USA, 1995.

[35] A. Edilson Camargo, M. Harold, H. Chong, and M. Richard De La Rue, "2D Photonic crystal thermo-optic switch based on AlGaAs/GaAs epitaxial structure," Optics Express, vol. 12, no. 4, 2004.

[36] R. Han-Youl, H. Jeong-Ki, and L. Yong-Hee, "Conditions of single guided mode in two-dimensional triangular photonic crystal slab waveguides," Journal of Applied Physics, vol. 88, no. 9, 2000.

[37] G. Burlak, Martínez-Sánchez, The Cherenkov emission in regular and random photonic crystals," Progress in Electromagnetics Research (PIER), vol. 47, pp. 77-86, 2006.

[38] I. Carusotto, M. Artoni, G. C. La Rocca et al., "Slow group velocity and Cherenkov radiation," Physical Review Letters, vol. 87, Article ID 064801, 2001. 